\title{
On the smoothness of dual windows for Gabor windows supported on $[1,1]$
}

\author{
Lemvig, Jakob; Nielsen, Kamilla Haahr
}

Published in:

Proceedings of 13th SampTA (Sampling Theory and Applications)

Publication date:

2019

Document Version

Publisher's PDF, also known as Version of record

Link back to DTU Orbit

Citation (APA):

Lemvig, J., \& Nielsen, K. H. (2019). On the smoothness of dual windows for Gabor windows supported on [1, 1]. In Proceedings of 13th SampTA (Sampling Theory and Applications)

\section{General rights}

Copyright and moral rights for the publications made accessible in the public portal are retained by the authors and/or other copyright owners and it is a condition of accessing publications that users recognise and abide by the legal requirements associated with these rights.

- Users may download and print one copy of any publication from the public portal for the purpose of private study or research.

- You may not further distribute the material or use it for any profit-making activity or commercial gain

- You may freely distribute the URL identifying the publication in the public portal

If you believe that this document breaches copyright please contact us providing details, and we will remove access to the work immediately and investigate your claim 


\section{On the smoothness of dual windows for Gabor windows supported on $[-1,1]$}

\author{
Jakob Lemvig \\ Technical University of Denmark \\ Department of Applied Mathematics \\ and Computer Science \\ Matematiktorvet 303B \\ 2800 Kgs. Lyngby, Denmark \\ E-mail: jakle@dtu.dk
}

\author{
Kamilla H. Nielsen \\ Technical University of Denmark \\ Department of Applied Mathematics \\ and Computer Science \\ Matematiktorvet 303B \\ 2800 Kgs. Lyngby, Denmark \\ E-mail: kamni@dtu.dk
}

\begin{abstract}
We study Gabor frames of the form $\left\{e^{2 \pi i b m} \cdot g(\cdot-a k)\right\}_{m, k \in \mathbb{Z}}$ generated by $n$ times differentiable windows $g$ that are non-zero on an open interval of length $L>0$ with translation parameter $a=L / 2$ and modulation parameter $b \in(0,2 / L)$. We first review recent explicit constructions of all dual windows with sufficiently short support by the authors. We then show that the obtainable smoothness of dual windows depends on the location of singularities of $g$. Our proof yields an explicit construction procedure of smooth dual windows once the singularities of $g$ avoid the specified locations.
\end{abstract}

\section{INTRODUCTION}

Series expansion of signals in $L^{2}(\mathbb{R})$ based on Gabor frames is a popular choice in time-frequency analysis. A Gabor system is a function system of the form

$$
\mathcal{G}(g, a, b):=\left\{e^{2 \pi i b m \cdot} g(\cdot-a k)\right\}_{m, k \in \mathbb{Z}},
$$

where $a, b>0$ and $g \in L^{2}(\mathbb{R})$, while a Gabor frame $\mathcal{G}(g, a, b)$ is a Gabor system for which there exists constants $A, B>0$ such that

$$
A\|f\|^{2} \leq \sum_{\eta \in \mathcal{G}(g, a, b)}|\langle f, \eta\rangle|^{2} \leq B\|f\|^{2}
$$

for all $f \in L^{2}(\mathbb{R})$. When the upper bound of (1) holds, the Gabor system is said to be a Bessel sequence. If $\mathcal{G}(g, a, b)$ satisfies (1), there exists at least one function $h \in L^{2}(\mathbb{R})$, called a dual window, such that $\mathcal{G}(h, a, b)$ is a Bessel sequence and

$$
f=\sum_{m, k \in \mathbb{Z}}\left\langle f, e^{2 \pi i b m \cdot} g(\cdot-a k)\right\rangle e^{2 \pi i b m \cdot h(\cdot-a k)}
$$

has unconditional $L^{2}$-convergence for all $f \in L^{2}(\mathbb{R})$. If furthermore $a b<1$, there exist infinitely many functions $h \in L^{2}(\mathbb{R})$ for which (2) holds.

We will assume the window $g \in C^{n}(\mathbb{R}), n \in \mathbb{Z}_{\geq 0} \cup$ $\{\infty\}$, is non-zero on an open interval $\left(x_{0}, x_{0}+L\right)$ and supp $g=\left[x_{0}, x_{0}+L\right]$ and consider Gabor systems $\left\{e^{2 \pi i b m} \cdot g(\cdot-a k)\right\}_{m, k \in \mathbb{Z}}$ with translation parameter $a=L / 2$ and modulation parameter $b \in(0,2 / L)$.
The choice $a=L / 2$ guarantees that each Fourierlike system $\left\{e^{2 \pi i b m} \cdot g\left(\cdot-a k_{0}\right)\right\}_{m \in \mathbb{Z}}$, where $k_{0} \in \mathbb{Z}$, has overlap of length $L / 2$ with two other Fourier-like systems (those associated with translation $k_{0} \pm 1$ ).

The programme of this paper is to find and construct "optimal" dual windows, in the sense of time- and frequency localization. More precisely, we require the dual windows $h$ to have compact support, i.e, perfect time localization, and then optimize over the smoothness of $h \in C^{n}(\mathbb{R})$ as this corresponds to decay in the frequency domain.

The classical method of constructing dual windows by painless non-orthonormal expansions [4, Theorem 2] is, for $a=L / 2$, restricted to the modulation range $b \in(0,1 / L]$. Here, we are interested in "large" modulation values in the range $b \in(1 / L, 2 / L)$ as this allows for Gabor systems of arbitrarily small redundancy $(a b)^{-1}$.

By dilation and translation of the Gabor system, we can without loss of generality assume that the translation parameter is $a=1$, the modulation parameter $b \in(0,1)$, and $\operatorname{supp} g=[-1,1]$. We then define the window classes:

$$
\begin{array}{r}
V_{+}^{n}(\mathbb{R})=\left\{f \in C^{n}(\mathbb{R}): \operatorname{supp} f=[-1,1]\right. \text { and } \\
|f(x)|>0 \text { for all } x \in(-1,1)\}
\end{array}
$$

for each $n \in \mathbb{Z}_{>0} \cup\{\infty\}$. By a result of Christensen, Kim, and Kim [1, Corollary 2.8], it follows that $\mathcal{G}(g, 1, b)$ with $g \in V_{+}^{0}(\mathbb{R})$ is a frame for $L^{2}(\mathbb{R})$ for any value of $b \in(0,1)$; in addition, a continuous and compactly supported dual window always exists. As the window classes are nested $V_{+}^{n+1}(\mathbb{R}) \subset V_{+}^{n}(\mathbb{R})$ for $n \in \mathbb{Z}_{\geq 0}$, this means that $\mathcal{G}(g, 1, b)$ is a frame for $L^{2}(\mathbb{R})$ for any $g \in V_{+}^{n}(\mathbb{R})$ and $b \in(0,1)$.

For each $n \in \mathbb{Z}_{\geq 0} \cup\{\infty\}$ and $g \in V_{+}^{n}(\mathbb{R})$ the two authors study in [6] explicit constructions of compactly supported dual windows. It is shown in [6] (see Theorem II.4) that given $g \in V_{+}^{n}(\mathbb{R})$ there are infinitely 
many compactly supported dual windows with the same smoothness, i.e., $h \in C^{n}(\mathbb{R})$, for each value of $b \in(0,1)$. In spite of the plethora of $C^{n}$-smooth dual windows, this smoothness is, in general, optimal; e.g., if $g \in V_{+}^{0}(\mathbb{R})$ is $C^{\infty}$ on $(-\infty, 0)$ and $(0, \infty)$, but with a simple discontinuity of $g^{\prime}(x)$ at $x=0$, then no dual window $h$ in $C^{1}(\mathbb{R})$ exists (see Theorem III.1).

The aim of this note is twofold: we first review the construction of dual windows from [6] in Section II. Section III is the main contribution: we show that in many cases, for $g \in V_{+}^{n}(\mathbb{R}) \backslash V_{+}^{n+1}(\mathbb{R})$, it is possible to go beyond $C^{n}$-smoothness for dual windows. In the main result, Theorem III.2, we show that if the singularities of $g \in V_{+}^{n}(\mathbb{R}) \backslash V_{+}^{n+1}(\mathbb{R})$ do not occur at certain points, depending on the modulation parameter $b$, then there exist dual windows $h$ with greater smoothness than $C^{n}$.

\section{DUAL WINDOWS WITH EQUAL SMOOTHNESS}

\section{A. Setup, notation, and dual windows}

For $g \in V_{+}^{n}(\mathbb{R})$, define $\psi: \mathbb{R} \rightarrow \mathbb{C}$ by

$$
\psi(x)=\frac{1}{\sum_{\ell \in \mathbb{Z}} g(x+\ell)} \quad \text { for } x \in \mathbb{R} .
$$

Note, that $\psi$ is a 1-periodic function uniformly bounded from below and above by positive constants. Furthermore, if $g$ has the partition of unity property $\sum_{\ell \in \mathbb{Z}} g(\cdot+\ell)=1$, then $\psi(x)=1$ for $x \in \mathbb{R}$. Assume that $b \in(0,1)$ and let $k_{\max } \in \mathbb{Z}_{\geq 0}$ be given as

$$
k_{\max }=\max \left\{k \in \mathbb{Z}_{\geq 0}: k<\frac{b}{1-b}\right\} .
$$

Consider $g \in V_{+}^{0}(\mathbb{R})$. Let $b \in \mathbb{R}$ and let $z:[0,1] \rightarrow$ $\mathbb{C}$ be any measurable function. We define the following auxiliary functions for each $k \in\left\{0,1, \ldots, k_{\max }\right\}$ :

$$
\begin{array}{r}
\eta_{k}(x-k)=[-g(x+1) z(x+1)+b \psi(x+1)] \\
\cdot(-1)^{k} \prod_{j=1}^{k} \frac{g(x+1+j(1 / b-1))}{g(x+j(1 / b-1))}
\end{array}
$$

for $x \in[-1,-k(1 / b-1)]$ and

$$
\begin{aligned}
\gamma_{k}(x+k)= & {[g(x-1) z(x)+b \psi(x)] } \\
& \cdot(-1)^{k} \prod_{j=1}^{k} \frac{g(x-1-j(1 / b-1))}{g(x-j(1 / b-1))}
\end{aligned}
$$

for $x \in[k(1 / b-1), 1]$. We then define $h_{z}: \mathbb{R} \rightarrow \mathbb{C}$ as

$$
\begin{aligned}
& \overline{h_{z}(x)}=\sum_{k=0}^{k_{\max }} \eta_{k}(x) \chi_{[-k-1,-k / b]}(x) \\
& +\sum_{k=0}^{k_{\max }} \gamma_{k}(x) \chi_{[k / b, k+1]}(x) \quad \text { for } x \in \mathbb{R} \backslash\{0\},
\end{aligned}
$$

and $\overline{h_{z}(0)}=b \psi(0)$.

The function $h_{z}$ is constructed in such a way that the window $g \in V_{+}^{0}(\mathbb{R})$ and $h_{z}$ defined by (7) satisfy the duality condition by Ron and Shen [7], [8] for any choice of the function $z$. This fact (shown in [6]) justifies the definition of $h_{z}$ and leads to the following result:

Theorem II.1 ([6]). Let $b \in(0,1)$, let $g \in V_{+}^{0}(\mathbb{R})$, and let $z:[0,1] \rightarrow \mathbb{C}$ be a measurable function. If $\mathcal{G}\left(h_{z}, 1, b\right)$ is a Bessel sequence, e.g., if $h_{z} \in L^{\infty}(\mathbb{R})$, then $\mathcal{G}(g, 1, b)$ and $\mathcal{G}\left(h_{z}, 1, b\right)$ are dual frames for $L^{2}(\mathbb{R})$.

By Theorem II.1, it is straightforward to construct compactly supported dual windows $h_{z}$ of any window $g \in V_{+}^{0}(\mathbb{R})$. In fact, the map $z \mapsto h_{z}$ parametrizes all dual windows with support in $\left[-k_{\max }-1, k_{\max }+1\right]$. In other words, any dual window $h$ with support $\left[-k_{\max }-1, k_{\max }+1\right]$ can be expressed as $h=h_{z}$ for some choice of $z$. In the next section we will relate properties of $h_{z}$ to properties of $z$. This will make it feasible to construct compactly supported dual windows $h_{z}$ with desirable properties.

\section{B. Properties of the dual window $h_{z}$}

To apply Theorem II.1 one needs the Bessel property of $\mathcal{G}\left(h_{z}, 1, b\right)$, which is guaranteed if, e.g., $h_{z} \in$ $L^{\infty}(\mathbb{R})$. The following result shows that boundedness of $h_{z}$ is equivalent to boundedness of $z$.

Lemma II.2 ([6]). Suppose $g \in V_{+}^{0}(\mathbb{R})$. Let $b \in(0,1)$. Then $z \in L^{\infty}(\mathbb{R})$ if and only if $h_{z} \in L^{\infty}(\mathbb{R})$.

A key property of the parametrization $z \mapsto h_{z}$ is that it allows for the construction of dual windows $h_{z} \in C^{n}(\mathbb{R})$ that are as smooth as the original window $g \in V_{+}^{n}(\mathbb{R})$. The smoothness conditions are given in Lemma II.3 and Theorem II.4 below; the given conditions are easy to satisfy and rely only on the function values of $z \in C^{n}([0,1])$ and its derivatives at the endpoints $x=0$ and $x=1$.

Lemma II.3 ([6]). Let $b \in(0,1)$. Suppose $g \in V_{+}^{0}(\mathbb{R})$. Then $h_{z} \in C^{0}(\mathbb{R})$ if and only if $z:[0,1] \rightarrow \mathbb{C}$ is a continuous function satisfying

$$
z(0)=\frac{b \psi(0)}{g(0)}=\frac{b}{g(0)^{2}}
$$

and

$$
z(1)=-\frac{b \psi(1)}{g(0)}=-\frac{b}{g(0)^{2}} .
$$

Note that if $g$ has the partition of unity property, i.e., $\sum_{\ell \in \mathbb{Z}} g(x+\ell)=1$ for all $x \in \mathbb{R}$, then $g(0)=1$ and the right hand sides simplify to $b$ and $-b$, respectively.

Theorem II.4 ([6]). Let $n \in \mathbb{Z}_{>0} \cup\{\infty\}$, and let $g \in V_{+}^{n}(\mathbb{R})$. The following assertions are equivalent: 
(i) $z \in C^{n}([0,1])$ satisfies (8), (9), and, for each $m=1, \ldots, n$, and $k=0,1$

$$
\begin{aligned}
z^{(m)}(k)= & -\sum_{\ell=1}^{m}\left(\begin{array}{c}
m \\
\ell
\end{array}\right) \frac{g^{(\ell)}(0)}{g(0)} z^{(m-\ell)}(k) \\
& +(-1)^{k} b \frac{\psi^{(m)}(0)}{g(0)},
\end{aligned}
$$

(ii) $h_{z} \in C^{n}(\mathbb{R})$.

If $g$ forms a partition of unity by integer translates, then, for each $m=1, \ldots, n$, condition (10) reduces to

$$
z^{(m)}(0)=z^{(m)}(1)=0 .
$$

III. DUAL WINDOWS WITH GREATER SMOOTHNESS

The next result is an obstruction result for the smoothness of dual windows. It shows that if the $(n+1)$ th derivative of the original window $g \in V_{+}^{n}(\mathbb{R})$ has a simple discontinuity at $x=0$, then the $C^{n}$. smoothness of dual windows is optimal.

Theorem III.1 ([6]). Let $b \in(0,1), h \in L^{2}(\mathbb{R})$ and $\operatorname{supp} g \subset[-1,1]$. For $n \in \mathbb{Z}_{\geq 0}$, we let $g \in C^{n}(\mathbb{R})$ be a piecewise $C^{n+1}$-function. For $n=-1$, we let $g \in L^{2}(\mathbb{R})$ be a piecewise continuous function. If $\mathcal{G}(g, 1, b)$ and $\mathcal{G}(h, 1, b)$ are dual frames and if $x=0$ is a discontinuity point of $g^{(n+1)}$ but $g^{(n+1)}$ is continuous at $x= \pm 1$ then $h \notin C^{n+1}(\mathbb{R})$.

As a consequence of Theorem III.1, if $g \in V_{+}^{n}(\mathbb{R})$, then, in general, one cannot expect dual windows $h \in C^{m}(\mathbb{R})$ for $m>n$. However, the main result of this note, Theorem III.2, exhibits a fairly large subclass of $g \in V_{+}^{n}(\mathbb{R})$ (given $b \in(0,1)$ ) for which the smoothness of the dual window can go beyond that of $g \in V_{+}^{n}(\mathbb{R})$. Note that Theorem III.2 assumes the partition of unity property of the original window $g$.

Theorem III.2. Let $b \in(0,1)$, let $n \in \mathbb{Z}_{>0} \cup\{\infty\}$, and let $g \in V_{+}^{0}(\mathbb{R})$ be a piecewise $C^{n}$-function satisfying $\sum_{\ell \in \mathbb{Z}} g(x+\ell)=1$ for all $x \in \mathbb{R}$. Let $\left\{x_{r} \in[-1,1]: r=1, \ldots, R\right\}$ denote the finite collection of points where $g^{(m)}$ for some $m=1, \ldots, n$ is discontinuous. Let

$$
\left\{t_{r}\right\}_{r=1}^{R}=\left\{x_{r}\right\}_{r=1}^{R} \bmod 1 .
$$

Define

$S_{n e g}=\bigcup_{k=1}^{k_{\max }}\left(\left\{t_{r}-k(1 / b-1)\right\}_{r=1}^{R}\right.$

$$
\cap[0,1-k(1 / b-1)]),
$$

and

$$
S_{\text {pos }}=\bigcup_{k=1}^{k_{\max }}\left(\left\{t_{r}+k(1 / b-1)\right\}_{r=1}^{R}\right.
$$

If

$$
t_{r} \neq 0, \quad r=1, \ldots, R
$$

and

$$
\left\{t_{r}\right\}_{r=1}^{R} \cap S_{\text {neg }} \cap S_{\text {pos }}=\emptyset,
$$

then there exists $h \in C^{n}(\mathbb{R})$ with compact support so that $\mathcal{G}(g, 1, b)$ and $\mathcal{G}(h, 1, b)$ are dual frames.

Remark 1. Before we prove Theorem III.2, let us provide some intuition behind the used construction. Let $\left\{t_{r}\right\}_{r=1}^{R}, S_{n e g}$ and $S_{p o s}$ be defined by (12), (13) and (14), respectively. The role of these three sets can be explained as follows. Assume for the sake of the argument that $z(x)=1$ for all $x$. The sets then describe all the possible discontinuities of $h_{z}$ and its derivatives up to order $n$. In particular, the set $\left\{t_{r}\right\}_{r=1}^{R}$ describes all the discontinuities that arise from $g(x)$ and $g(x-1)$. The sets $S_{n e g}$ and $S_{p o s}$ describe all the discontinuities that arise from $\prod_{j=1}^{k} \frac{g(x+j(1 / b-1))}{g(x-1+j(1 / b-1))}$ for $x \in[0,1-k(1 / b-1)], k=1, \ldots, k_{\max }$ and $\prod_{j=1}^{k} \frac{g(x-1-j(1 / b-1))}{g(x-j(1 / b-1))}$ for $x \in[k(1 / b-1), 1], k=$ $1, \ldots, k_{\max }$, respectively.

Proof of Theorem III.2. The proof is based on the parametrization of all dual windows $z \mapsto h_{z}$ and the following simple fact. If $u$ is a piecewise $C^{n}$ function with a discontinuity at $x_{0}$ and $v \in C^{n}(\mathbb{R})$ with $v^{(m)}\left(x_{0}\right)=0$ for $m=0,1, \ldots, n$, then $u v$ is $C^{n}$ in a neighborhood of $x_{0}$ and $(u v)^{(m)}\left(x_{0}\right)=0$ for $m=0,1, \ldots, n$.

To obtain a smooth dual, we must choose the $z$ function such that it smooths out the discontinuities created by the $g$ window in the construction of $h_{z}$. Hence, to obtain a dual window $h_{z} \in C^{n}(\mathbb{R})$, it suffices to take $z:[0,1] \rightarrow \mathbb{C}$ as a $C^{n}$-function satisfying (8), (9), and (11) as well as

$$
z^{(m)}(x)=0
$$

for all $m=0,1, \ldots, n$ and $x \in\left\{t_{r}\right\}_{r=1}^{R}$,

$$
\frac{d^{m}}{d x^{m}}(-g(x) z(x)+b)=0
$$

for all $m=0,1, \ldots, n$ and $x \in S_{n e g}$, and

$$
\frac{d^{m}}{d x^{m}}(g(x-1) z(x)+b)=0
$$

for all $m=0,1, \ldots, n$ and $x \in S_{p o s}$. Note that $z^{(0)}(x)=z(x)=0$ for $x \in\left\{t_{r}\right\}_{r=1}^{R}$ does not violate (8) and (9) as 0 does not belong to $\left\{t_{r}\right\}_{r=1}^{R}$ due to assumption (15). Furthermore, none of the conditions (17), (18) or (19) violate each other due to assumption (16).

The conditions (17), (18) or (19) are formulated with the relation to $h_{z}$ in mind. However, for construction of dual windows we need to specify $z$, and it is, 
therefore, more convenient to consider the equations with $z^{(m)}$ being isolated. Now, under the assumption of Theorem III.2, we have a dual window $h_{z} \in C^{n}(\mathbb{R})$, $n \in \mathbb{Z}_{>0} \cup\{\infty\}$, if $z$ satisfies (8), (9), (11),

$$
z(x)= \begin{cases}0, & x \in\left\{t_{r}\right\}_{r=1}^{R}, \\ \frac{b}{g(x)}, & x \in S_{\text {neg }}, \\ -\frac{b}{g(x-1)}, & x \in S_{\text {pos }},\end{cases}
$$

and the following conditions for each $m=1, \ldots, n$ :

$$
\begin{aligned}
& z^{(m)}(x)=0 \quad \text { for all } x \in\left\{t_{r}\right\}_{r=1}^{R}, \\
& z^{(m)}(x)=-\sum_{\ell=1}^{m}\left(\begin{array}{c}
m \\
\ell
\end{array}\right) \frac{g^{(\ell)}(x)}{g(x)} z^{(m-\ell)}(x)
\end{aligned}
$$

for all $x \in S_{n e g}$, and

$$
z^{(m)}(x)=-\sum_{\ell=1}^{m}\left(\begin{array}{c}
m \\
\ell
\end{array}\right) \frac{g^{(\ell)}(x-1)}{g(x-1)} z^{(m-\ell)}(x)
$$

for all $x \in S_{\text {pos }}$.

Example 1. Let $b \in(0,1)$, and let $g \in V_{+}^{0}(\mathbb{R})$ be a $C^{\infty}$-function except at a single point $x \notin\{-1,0,1\}$, where $g^{\prime}(x)$ fails to be continuous. Assume also that $\sum_{\ell \in \mathbb{Z}} g(x+\ell)=1$ for all $x \in \mathbb{R}$. Despite the lack of smoothness of $g \notin C^{1}(\mathbb{R})$, we can, by Theorem III.2, construct dual windows $h \in C^{n}(\mathbb{R})$ with compact support for any $n \in \mathbb{Z}_{\geq 0}$ or even $n=\infty$. More concretely, we take $z$ to be a $C^{n}$-function satisfying the conditions (8), (9), (11), and (20)-(23).

Example 2. Let $b=\frac{3}{5}$ and let $g \in V_{+}^{0}(\mathbb{R})$ be piecewise $C^{\infty}$-function with simple discontinuities of $g^{\prime}(x)$ at $\left\{x_{r}\right\}_{r=1}^{4}=\left\{-\frac{3}{4},-\frac{1}{5}, \frac{1}{4}, \frac{4}{5}\right\}$, and assume $g$ satisfies $\sum_{\ell \in \mathbb{Z}} g(\cdot+\ell)=1$. Since $g \in V_{+}^{0}(\mathbb{R})$ the Gabor system $\mathcal{G}(g, 1, b)$ is a frame for $L^{2}(\mathbb{R})$. We first calculate $k_{\text {max }}=1$ and the three sets:

$$
\begin{gathered}
\left\{t_{r}\right\}_{r=1}^{4}=\left\{\frac{1}{4}, \frac{4}{5}, \frac{1}{4}, \frac{4}{5}\right\}, \\
S_{n e g}=\left\{\frac{2}{15}\right\} \text { and } S_{p o s}=\left\{\frac{11}{12}\right\} .
\end{gathered}
$$

Since these sets satisfy the conditions (15) and (16), we can apply Theorem III. 2 to construct a compactly supported dual window $h \in C^{n}(\mathbb{R})$ for any value of $n \in \mathbb{Z}_{\geq 0} \cup\{\infty\}$. As above we simply need $z$ to satisfy (8), (9), (11), and (20)-(23)

Let us here illustrate the construction for $n=1$. We have $h_{z} \in C^{1}(\mathbb{R})$ if a function $z \in C^{1}(\mathbb{R})$ satisfies

$$
z(x)= \begin{cases}b, & x=0, \\ \frac{b}{g\left(\frac{2}{15}\right)}, & x=\frac{2}{15}, \\ 0, & x \in\left\{\frac{1}{4}, \frac{4}{5}\right\}, \\ -\frac{b}{g\left(-\frac{1}{12}\right)}, & x=\frac{11}{12}, \\ -b, & x=1\end{cases}
$$

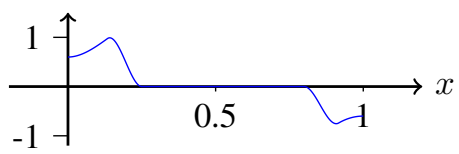

Figure 1. The spline function $z \in C^{1}(\mathbb{R})$ from Example 2 . It is a piecewise polynomial of degree 3 with knots $x=$ $0,2 / 15,1 / 4,4 / 5,11 / 12,1$.

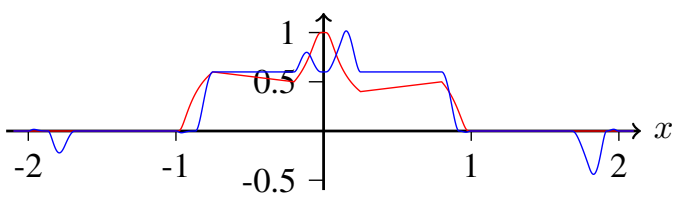

Figure 2. The window $g \in V_{+}^{0}(\mathbb{R}) \backslash V_{+}^{1}(\mathbb{R})$ (red) and a dual window $h_{z} \in C^{1}(\mathbb{R})$ (blue) corresponding to $z$ given as in Figure 1 .

and

$$
z^{\prime}(x)= \begin{cases}0, & x \in\left\{0, \frac{1}{4}, \frac{4}{5}, 1\right\}, \\ -b g^{\prime}\left(\frac{2}{15}\right) / g\left(\frac{2}{15}\right)^{2}, & x=\frac{2}{15}, \\ b g^{\prime}\left(-\frac{1}{12}\right) / g\left(-\frac{1}{12}\right)^{2}, & x=\frac{11}{12} .\end{cases}
$$

We take $z \in C^{1}(\mathbb{R})$ to be the unique degree 3 piecewise polynomial with knots at $x=$ $0,2 / 15,1 / 4,4 / 5,11 / 12,1$ that satisfies the above 12 conditions, see Figure 1.

The window $g \in V_{+}^{0}(\mathbb{R}) \backslash V_{+}^{1}(\mathbb{R})$ and the corresponding dual window $h_{z} \in C^{1}(\mathbb{R})$ with support $\operatorname{supp} h_{z}=\left[-2,-\frac{5}{3}\right] \cup[-1,1] \cup\left[\frac{5}{3}, 2\right]$ are shown in Figure 2.

\section{REFERENCES}

[1] O. Christensen, H. O. Kim, and R. Y. Kim. Gabor windows supported on $[-1,1]$ and compactly supported dual windows. Appl. Comput. Harmon. Anal., 28(1):89-103, 2010. URL: http://dx.doi.org/10.1016/j.acha.2009.07.004.

[2] O. Christensen, H. O. Kim, and R. Y. Kim. Gabor windows supported on $[-1,1]$ and dual windows with small support. Adv. Comput. Math., 36(4):525-545, 2012. URL: http://dx.doi.org/10.1007/s10444-011-9189-0.

[3] O. Christensen. An introduction to frames and Riesz bases. Applied and Numerical Harmonic Analysis. Birkhäuser Boston Inc., Boston, MA, 2003.

[4] I. Daubechies, A. Grossmann, and Y. Meyer. Painless nonorthogonal expansions. J. Math. Phys., 27(5):1271-1283, 1986. URL: http://dx.doi.org/10.1063/1.527388.

[5] K. Gröchenig. Foundations of time-frequency analysis. Applied and Numerical Harmonic Analysis. Birkhäuser Boston, Inc., Boston, MA, 2001.

[6] J. Lemvig and K. H. Nielsen. Gabor windows supported on $[-1,1]$ and construction of compactly supported dual windows with optimal frequency localization, preprint.

[7] A. Ron and Z. Shen. Frames and stable bases for shift-invariant subspaces of $L_{2}\left(\mathbf{R}^{d}\right)$. Canad. J. Math., 47(5):1051-1094, 1995. URL: http://dx.doi.org/10.4153/CJM-1995-056-1.

[8] A. Ron and Z. Shen. Weyl-Heisenberg frames and Riesz bases in $L_{2}\left(\mathbf{R}^{d}\right)$. Duke Math. J., 89(2):237-282, 1997. URL: http://dx.doi.org/10.1215/S0012-7094-97-08913-4. 